\title{
Selective spatial damping of propagating kink waves due to resonant absorption
}

\author{
J. Terradas ${ }^{1}$, M. Goossens ${ }^{2}$, and G. Verth ${ }^{2}$ \\ 1 Departament de Física, Universitat de les Illes Balears, Spain \\ e-mail: jaume.terradas@uib.es \\ 2 Centre Plasma Astrophysics and Leuven Mathematical Modeling and Computational Science Centre, \\ Katholieke Universiteit Leuven, Leuven, 3001, Belgium \\ e-mail: [Marcel.Goossens; Gary.Verth]@wis . kuleuven. be
}

Received 22 April 2010 / Accepted 11 August 2010

\begin{abstract}
Context. There is observational evidence of propagating kink waves driven by photospheric motions. These disturbances, interpreted as kink magnetohydrodynamic (MHD) waves are attenuated as they propagate upwards in the solar corona.

Aims. We show that resonant absorption provides a simple explanation to the spatial damping of these waves.

Methods. Kink MHD waves are studied using a cylindrical model of solar magnetic flux tubes, which includes a non-uniform layer at the tube boundary. Assuming that the frequency is real and the longitudinal wavenumber complex, the damping length and damping per wavelength produced by resonant absorption are analytically calculated in the thin tube (TT) approximation, valid for coronal waves. This assumption is relaxed in the case of chromospheric tube waves and filament thread waves.

Results. The damping length of propagating kink waves due to resonant absorption is a monotonically decreasing function of frequency. For kink waves with low frequencies, the damping length is exactly inversely proportional to frequency, and we denote this as the TGV relation. When moving to high frequencies, the TGV relation continues to be an exceptionally good approximation of the actual dependency of the damping length on frequency. This dependency means that resonant absorption is selective as it favours lowfrequency waves and can efficiently remove high-frequency waves from a broad band spectrum of kink waves. The efficiency of the damping due to resonant absorption depends on the properties of the equilibrium model, in particular on the width of the non-uniform layer and the steepness of the variation in the local Alfvén speed.

Conclusions. Resonant absorption is an effective mechanism for the spatial damping of propagating kink waves. It is selective because the damping length is inversely proportional to frequency so that the damping becomes more severe with increasing frequency. This means that radial inhomogeneity can cause solar waveguides to be a natural low-pass filter for broadband disturbances. Kink wave trains travelling along, e.g., coronal loops, will therefore have a greater proportion of the high-frequency components dissipated lower down in the atmosphere. This could have important consequences for the spatial distribution of wave heating in the solar atmosphere.
\end{abstract}

Key words. magnetohydrodynamics - waves - magnetic fields - Sun: atmosphere - Sun: oscillations

\section{Introduction}

The first observations of post-flare transversal coronal loop oscillations by the transition region and coronal explorer (TRACE) (e.g., Aschwanden et al. 1999; Nakariakov et al. 1999; Aschwanden et al. 2002), have inspired much development in magnetohydrodynamic (MHD) wave theory. This observational breakthrough was important since estimated wave parameters, such as frequency and amplitude have allowed us to implement magnetoseismological techniques to probe the plasma fine structure of the Sun's atmosphere, an idea initially proposed by, e.g., Uchida (1970) and Roberts et al. (1984). It is now commonly accepted that these transversal waves are the kink mode from MHD wave theory (see e.g., Edwin \& Roberts 1983), a highly magnetically dominated Alfvénic wave (see Goossens et al. 2009, for a discussion on the nature of kink waves). The observed post-flare kink waves in coronal loops have two main defining characteristics; firstly, they are standing modes, and secondly, they are strongly damped oscillations (in about 1-4 periods, see e.g., Aschwanden et al. 2003). Initially there were several physical mechanisms proposed to explain the observed damping, e.g., footpoint leakage (Berghmans \& de Bruyne 1995;
De Pontieu et al. 2001), lateral wave leakage (Smith et al. 1997; Brady \& Arber 2005; Verwichte et al. 2006; Selwa et al. 2005, 2007; McLaughlin \& Ofman 2008), phase mixing (Heyvaerts \& Priest 1983; Roberts 2002; Ofman \& Aschwanden 2002), resonant absorption (Ruderman \& Roberts 2002; Goossens et al. 2002), and more recently loop cooling (Morton \& Erdélyi 2009). Thus far, resonant absorption, caused by plasma inhomogeneity in the direction transverse to the magnetic field (see Ionson 1978; Hollweg \& Yang 1988; Steinolfson \& Davila 1993; Ofman \& Davila 1995), has proved the most likely candidate for explaining the observed short damping times in coronal loops (see Goossens 2008, for review).

Consistent seismological studies based on resonant absorption using the observed values of periods and damping times of standing kink waves were carried out by Arregui et al. (2007) and Goossens et al. (2008). These two studies show that, at least for a collection of 11 loops, resonant absorption provides an explanation of the observed damping times. The observational signatures of the alternative cooling loop damping mechanism proposed by Morton \& Erdélyi (2009) differ from resonant absorption by the fact that the frequency changes as a function of time. The resonant damping theory developed so far is restricted 
to static and stationary equilibria, and therefore frequency is expected to be constant in time. Extensive MHD modelling has also shown that resonant absorption is the most likely explanation for the damping of transverse oscillations in fine structures of prominences (see Arregui et al. 2008; Soler et al. 2009a,b; Arregui \& Ballester 2010).

If indeed, coronal loop kink oscillations are being attenuated by the process of resonant absorption, one can exploit this to estimate the transverse plasma inhomogeneity length scales using observed frequencies and damping times (Goossens et al. 2006). The original equilibrium models by, e.g., Ruderman \& Roberts (2002), for studying resonant absorption consisted of monolithic loop structures. However, there has been some observational evidence by, e.g., Aschwanden (2005) that coronal loops are composed of many different strands, possibly at different temperatures and densities. To model this loop multi-thread structure, Terradas et al. (2008) has numerically solved the initial value problem and found that the process of resonant absorption was still an efficient damping mechanism in more complex and realistically structured loop models (see also the work of Ofman 2005). Further study into the properties of standing kink waves has also been undertaken relating plasma inhomogeneity in the direction of the magnetic field caused by, e.g., density (Díaz et al. 2004; Andries et al. 2005b; Arregui et al. 2005; Dymova \& Ruderman 2006; Erdélyi \& Verth 2007; Verth et al. 2007) or magnetic (Verth \& Erdélyi 2008; Ruderman et al. 2008) stratification. It was shown that eigenfrequencies and eigenfunctions of coronal loops with longitudinal stratification were altered in such a way that one could determine, e.g., the coronal density scale height by estimating the ratio of the fundamental mode to that of higher overtones, a technique first proposed by Andries et al. (2005a) and later developed by Verth et al. (2008) to correct for magnetic stratification.

All these theoretical developments to describe kink waves in coronal loops with realistic plasma stratification in the transverse and longitudinal direction were restricted to studying standing waves, since this was what was detected in TRACE data. However, recently it has come to light that there are also ubiquitous small-amplitude, propagating transversal MHD waves in the solar atmosphere. These were first observed by the novel Coronal Multi-channel Polarimeter (CoMP) instrument (Tomczyk et al. 2007). Moreover, Tomczyk \& McIntosh (2009) have been able to separate outward and inward propagating wave power. It was found that the outward power was greater than the inward power by about a factor of two, and this can only be explained if the waves are damped in situ (see also Pascoe et al. 2010). The reason we could not detect these propagating waves previously with TRACE is that the amplitudes are of the order of $50 \mathrm{~km}$, while the TRACE resolution is only about $800 \mathrm{~km}$. Tomczyk et al. (2007) originally interpreted these wave as Alfvén waves, but Van Doorsselaere et al. (2008) subsequently argued that the observed waves were actually more consistent with the propagating kink mode. Although both modes are dominated by the restoring force of magnetic tension, in the geometry of a solar flux tube, e.g., a magnetic cylinder, a pure Alfvén wave is strictly torsional with no transverse component and therefore completely incompressible. On the other hand, a kink wave propagating in a flux tube has a transverse perturbation component and is weakly compressible, at least in the linear regime (see e.g., Goossens et al. 2009).

In the present paper, we restrict the study to investigating the effect of transverse plasma inhomogeneity on the propagating kink mode. Although the two problems of standing and propagating kink waves are closely related, since a standing wave is a superposition of two propagating waves, there are some differences that need to be considered. The standing transverse oscillation is the result of an initial value problem, i.e., an initial disturbance in the solar corona such as a CME or a flare, induces the oscillations of the loops at their natural frequencies or eigenmodes. Alternately, the transverse travelling waves have a forced nature since the photosphere acts as a driver. The frequencies of the kink waves observed by CoMP show a peak around $5 \mathrm{~min}$, indicating a $p$-mode-driven photospheric origin. The spatial scale of the driver at the base of the tube is also important in exciting kink oscillations. From the properties of MHD waves in a flux tube, we know that a purely incompressible excitation excites purely incompressible Alfvén waves if the driver is strictly localised inside or outside the tube (assuming a homogeneous loop model). On the other hand, an excitation located both inside and outside the tube invariably excites transverse oscillations since an almost incompressible surface wave between the two media will be established, i.e., the kink mode.

\section{Waveguide model}

To understand the effect of radial inhomogeneity on propagating kink waves we consider the relatively simple equilibrium model of a cylindrical axi-symmetric flux tube of radius $R$ with a constant axial magnetic field $B_{z}$ and with a density contrast of $\rho_{\mathrm{i}} / \rho_{\mathrm{e}}$. The subindexes " $\mathrm{i}$ " and "e" refer to the internal and external part of the tube, respectively. It is also assumed that the tube has a smooth variation in density across the waveguide boundary (located at $r=R$ ) on a characteristic spatial scale $l$. For simplicity, a sinusoidal density profile connecting the internal and external part of the tube is implemented (see e.g., Ruderman \& Roberts 2002; Goossens et al. 2002; Terradas et al. 2006). The role of the inhomogeneity at the loop boundary is crucial since this is where the process of resonant absorption invariably takes place. This basically means that the transverse displacement of the whole tube is converted into azimuthal motions localised at the tube boundary. The transverse motion is attenuated by this energy conversion, while at the same time small scales are created in the nonuniform layer from phase mixing. Phase mixing causes a cascade of energy to smaller length scales, where the dissipation becomes more efficient. The reader is referred to Goossens (2008), Ruderman \& Erdélyi (2009), Terradas (2009), and references therein for further details about this robust damping mechanism. Recent studies by Soler et al. (2009a) in the context of modelling kink oscillations observed in solar prominences (complex coronal magnetic structures with relatively cool and dense plasma) show that the process of resonant absorption still survives even when the plasma is partially ionised.

\section{Spatial damping in the TT approximation}

Before we embark on analysing MHD waves in the thin tube (TT) approximation, let us explain what this approximation actually is. In what follows we consider both standing and propagating waves. A standing wave is the superposition of two propagating waves travelling with the same frequency and wavenumber but in opposite directions. For the standing wave problem the axial wavelength (or the axial wavenumber $k_{z}$ ) is specified and the corresponding frequency is determined. A TT in this case means that the axial wavelength is much longer than the radius of the tube so that $k_{z} R \ll 1$, so the TT approximation for standing waves is the long wavelength approximation. The frequency $\omega$ is specified for propagating waves and the corresponding wavelength determined. In this situation, a TT means that during one 
period, defined by the frequency $\omega$, a signal travelling at the Alfvén speed can cross the waveguide in the radial direction many times or $\omega /\left(v_{\mathrm{A}} / R\right) \ll 1$. As a result the TT approximation is the low-frequency approximation for propagating waves. The benefit of the TT approximation is that it enables us to obtain simple mathematical expressions that are very accurate, allowing us to gain a physical insight into the problem.

\subsection{Homogeneous magnetic cylinder}

For a homogeneous magnetic cylinder (i.e., no inhomogeneous layer, $l=0)$, the dispersion relation for the kink mode $(m=1)$ and the fluting modes $(m>1)$ in the TT approximation is (see Goossens et al. 2009)

$\rho_{\mathrm{i}}\left(\omega^{2}-\omega_{\mathrm{Ai}}^{2}\right)+\rho_{\mathrm{e}}\left(\omega^{2}-\omega_{\mathrm{Ae}}^{2}\right)=0$,

where

$\omega_{\mathrm{A}}^{2}=k_{z}^{2} v_{\mathrm{A}}^{2}, \quad v_{\mathrm{A}}^{2}=B_{z}^{2} /(\mu \rho)$.

The dispersion relation Eq. (1) specifies a functional dependence on frequency $\omega$ and wavenumber $k_{z}$. This can be studied for the cases of either standing or propagating waves. For the propagating wave study, the wavenumber $k_{z}$ is specified and the dispersion relation is solved for frequency $\omega$, leading to the well known result

$\omega^{2}=\frac{\rho_{\mathrm{i}} \omega_{\mathrm{Ai}}^{2}+\rho_{\mathrm{e}} \omega_{\mathrm{Ae}}^{2}}{\rho_{\mathrm{i}}+\rho_{\mathrm{e}}} \equiv \omega_{*}^{2}$,

where $\omega_{*}$ is real. For equal magnetic field strength inside and outside the cylinder this expression can be further simplified to

$\omega^{2}=\frac{2 B_{z}^{2}}{\mu\left(\rho_{\mathrm{i}}+\rho_{\mathrm{e}}\right)} k_{z}^{2}=\frac{2 \rho_{\mathrm{i}}}{\rho_{\mathrm{i}}+\rho_{\mathrm{e}}} v_{\mathrm{Ai}}^{2} k_{z}^{2} \equiv \omega_{*}^{2}$.

For propagating waves we consider waves generated at a given location with a real frequency $\omega_{*}$ and solve the dispersion relation for $k_{z}$, resulting in

$k_{z}^{2}=\frac{\mu\left(\rho_{\mathrm{i}}+\rho_{\mathrm{e}}\right)}{2 B_{z}^{2}} \omega_{*}^{2}=\frac{\rho_{\mathrm{i}}+\rho_{\mathrm{e}}}{2 \rho_{\mathrm{i}}} \frac{\omega_{*}^{2}}{v_{\mathrm{Ai}}^{2}} \equiv k_{*}^{2}$,

where $k_{*}$ is also real. The solution given by Eq. (5) corresponds to the well known undamped kink wave (and the fluting modes). To derive Eq. (1) it is implicitly assumed that $\omega R / v_{\mathrm{A}} \ll 1$, but since not all frequencies will satisfy these conditions later, we present results for any frequency of driver without this restriction.

\subsection{Inhomogeneous magnetic cylinder}

The thin boundary (TB) approximation means that the nonuniformity is confined to $[R-l / 2, R+l / 2]$, with $l / R \ll 1$, so that the non-uniform layer coincides with the dissipative layer. This approximation results in the mathematical simplification that MHD waves can be described by solutions for uniform plasmas that are connected over the dissipative layer by jump conditions (see e.g., Sakurai et al. 1991; Goossens et al. 1995; Tirry \& Goossens 1996). The use of the TB approximation has been applied in e.g., Goossens et al. (2006, 2009) and Goossens (2008). Including the effect of an inhomogeneous layer is reasonably simple in the case of the thin boundary approximation and results in the following dispersion relation,

$$
\begin{aligned}
\rho_{\mathrm{i}}\left(\omega^{2}-\omega_{\mathrm{Ai}}^{2}\right)+ & \rho_{\mathrm{e}}\left(\omega^{2}-\omega_{\mathrm{Ae}}^{2}\right)= \\
& \mathrm{i} \pi \frac{m / r_{\mathrm{A}}}{\rho\left(r_{\mathrm{A}}\right)\left|\Delta_{\mathrm{A}}\right|} \rho_{\mathrm{i}}\left(\omega^{2}-\omega_{\mathrm{Ai}}^{2}\right) \rho_{\mathrm{e}}\left(\omega^{2}-\omega_{\mathrm{Ae}}^{2}\right) .
\end{aligned}
$$

Here $r_{\mathrm{A}}$ denotes the position of the Alfvén resonance. In the TB approximation it is natural to adopt $r_{\mathrm{A}}=R$ since $l / R \ll 1$ and $\left.r_{\mathrm{A}} \in\right] R-l / 2, R+l / 2$ [. Using the jump condition is not restricted to the thin non-uniform layer as can be seen from, e.g., Tirry \& Goossens (1996); Tirry et al. (1997, 1998); however, this condition requires numerical integration of the ideal MHD equations in a non-uniform plasma up to the dissipative layer. In the present paper we do not intend to use numerical integration of the ideal MHD equations relating to thick boundaries. However, we do use the results obtained with the TB approximation for thick boundaries for a comparison with the results of a full numerical calculation in Sect. 5.

The effect of resonance is contained on the right hand side of Eq. (6). Again we can view the dispersion relation Eq. (6) as a relation for either standing or propagating waves. In the case of standing waves, the wavenumber is real $\left(k_{z}=k_{*}\right)$ and the frequency is complex. This case has been considered previously by e.g., Goossens et al. (1992, 2009). Let us now focus on propagating waves with a given real frequency $\left(\omega=\omega_{*}\right)$ and complex wavenumber. The imaginary part of the wavenumber indicates that the wave, as it propagates, is damped by resonant absorption. Now we assume that

$k_{z}=k_{\mathrm{R}}+\mathrm{i} k_{\mathrm{I}}$.

The purely imaginary term in Eq. (7) reflects the damping imposed on the wave, and since the damping is in the spatial domain, the wavenumber is now complex. If we approximate $k_{z}^{2}$ by $k_{\mathrm{R}}^{2}+2 \mathrm{i} k_{\mathrm{R}} k_{\mathrm{I}}$ (we assume weak damping, i.e., $k_{\mathrm{I}} \ll k_{\mathrm{R}}$ ), we have that $k_{\mathrm{R}} \approx k_{*}$ (given by Eq. (5)), and after some algebra we find

$\frac{k_{\mathrm{I}}}{k_{*}}=\frac{\pi}{8} \frac{m}{R} \frac{1}{\rho\left(r_{\mathrm{A}}\right)\left|\Delta_{\mathrm{A}}\right|} \frac{\left(\rho_{\mathrm{i}}-\rho_{\mathrm{e}}\right)^{2}}{\left(\rho_{\mathrm{i}}+\rho_{\mathrm{e}}\right)} \omega_{*}^{2}$.

Because

$\rho\left(r_{\mathrm{A}}\right)\left|\Delta_{\mathrm{A}}\right|=\omega^{2}\left|\frac{\mathrm{d} \rho}{\mathrm{d} r}\right|_{r_{\mathrm{A}}}$,

we finally obtain the following expression

$\frac{k_{\mathrm{I}}}{k_{*}}=\frac{\pi}{8} \frac{m}{R} \frac{\left(\rho_{\mathrm{i}}-\rho_{\mathrm{e}}\right)^{2}}{\rho_{\mathrm{i}}+\rho_{\mathrm{e}}} \frac{1}{|\mathrm{~d} \rho / \mathrm{d} r|_{r_{\mathrm{A}}}}$.

Because $k_{z}$ is complex, we define the damping length as $L_{\mathrm{D}}=$ $1 / k_{\mathrm{I}}$, while the wavelength is simply $\lambda=2 \pi / k_{*}$. A useful quantity is the the damping per wavelength, which is

$\frac{L_{\mathrm{D}}}{\lambda}=\frac{4}{\pi^{2}} \frac{R}{m} \frac{\rho_{\mathrm{i}}+\rho_{\mathrm{e}}}{\left(\rho_{\mathrm{i}}-\rho_{\mathrm{e}}\right)^{2}}\left|\frac{\mathrm{d} \rho}{\mathrm{d} r}\right|_{r_{\mathrm{A}}}$.

For a sinusoidal density profile it can be shown that

$\left|\frac{\mathrm{d} \rho}{\mathrm{d} r}\right|_{r_{\mathrm{A}}}=\frac{\pi}{2} \frac{\rho_{\mathrm{i}}-\rho_{\mathrm{e}}}{l}$,

and Eq. (11) reduces to

$\frac{L_{\mathrm{D}}}{\lambda}=\frac{2}{\pi} \frac{1}{m} \frac{R}{l} \frac{\rho_{\mathrm{i}}+\rho_{\mathrm{e}}}{\rho_{\mathrm{i}}-\rho_{\mathrm{e}}}$. 
Equation (13) clearly shows the dependence of the damping per wavelength on the thickness of the layer. The wider the layer, the stronger the spatial attenuation of the wave. This is not surprising, since we can relate this result to the expression for the temporal damping, i.e., wavenumber assumed real $\left(k_{z}=k_{*}\right)$ and frequency complex. The well known formula in the case of the temporal damping for a standing wave is

$\frac{\tau_{\mathrm{D}}}{P}=\frac{2}{\pi} \frac{1}{m} \frac{R}{l} \frac{\rho_{\mathrm{i}}+\rho_{\mathrm{e}}}{\rho_{\mathrm{i}}-\rho_{\mathrm{e}}}$.

If we compare this expression with Eq. (13), we note that the damping per wavelength for propagating waves and the damping per period for standing waves are exactly the same so spatial and temporal damping are completely equivalent in the TT approximation. From Eqs. (13) and (14) we obtain the simple result

$\frac{L_{\mathrm{D}}}{\lambda}=\frac{\tau_{\mathrm{D}}}{P}$.

Propagating kink waves have been recently studied by Vasheghani Farahani et al. (2009) in the long-wavelength limit in X-ray jets in the solar atmosphere. These authors computed the ratio of the damping time to the period for standing waves and use this quantity to discuss the spatial damping of propagating waves. The TT approximation result presented in Eq. (15) therefore validates the use of the damping expression derived for kink standing waves by Vasheghani Farahani et al. (2009) to interpret the attenuation of propagating kink waves. The TT damping relations given by Eqs. (13) and (14) have other significant consequences. For standing waves, we rewrite Eq. (14) as

$\frac{\tau_{\mathrm{D}}}{P}=\frac{\xi_{\mathrm{E}}}{m}$

where

$\xi_{\mathrm{E}}=\frac{2}{\pi} \frac{R}{l} \frac{\rho_{\mathrm{i}}+\rho_{\mathrm{e}}}{\rho_{\mathrm{i}}-\rho_{\mathrm{e}}}$

which only depends on the parameters of the equilibrium model, not on the particular type of MHD wave mode defined by the value of $m$. For the kink mode $m=1$. The period is defined by

$P=\frac{2 \pi}{\omega}$,

and for standing waves $\omega$ is related to the wavenumber $k_{z}$ by the dispersion relation given by Eq. (4); i.e.,

$\omega=n v_{\mathrm{Ai}} \frac{\pi}{L} \sqrt{\frac{2 \rho_{\mathrm{i}}}{\rho_{\mathrm{i}}+\rho_{\mathrm{e}}}}$,

where $v_{\mathrm{Ai}}$ is the internal Alfvén speed, $n=1,2,3, \ldots$ is the longitudinal mode number, and $L$ is the total length of the waveguide, e.g., a coronal loop (we have used that $k_{z}=n \pi / L$ ). Equation (16) can then be written as

$\frac{\tau_{\mathrm{D}}}{\tau_{\mathrm{Ai}}}=\frac{2 \xi_{\mathrm{E}}}{m} \sqrt{\frac{\rho_{\mathrm{i}}+\rho_{\mathrm{e}}}{2 \rho_{\mathrm{i}}}} \frac{1}{n}$,

where $\tau_{\mathrm{Ai}}=L / v_{\mathrm{Ai}}$ is the Alfvén transit time in the longitudinal direction. Equation (20) has interesting consequences. Firstly, it indicates that fluting modes $(m>1)$ have shorter damping times than the kink mode $(m=1)$. Secondly, it shows that the damping time for a standing wave is inversely proportional to the longitudinal mode number $n$; i.e., the damping time is inversely proportional to the wavelength of the standing wave, so that higher overtones (with shorter periods) are damped faster than low-order overtones, e.g, the fundamental mode. Fortunately, there have been some signatures of overtones in coronal loop standing kink waves detected in TRACE data (see e.g. Verwichte et al. 2004; De Moortel \& Brady 2007; Verth et al. 2008; Van Doorsselaere et al. 2009). For a particularly clear example of the first overtone damping before the fundamental mode, that could be explained by resonant absorption attenuating the higher harmonic faster, see the Morlet wavelet transform in Fig. 5 of Verwichte et al. (2004).

Now considering the spatial damping of propagating waves, we can also write Eq. (13) as

$\frac{L_{\mathrm{D}}}{\lambda}=\frac{\xi_{\mathrm{E}}}{m}$.

The wavelength is defined as $\lambda=2 \pi / k_{z}$ and is related to the frequency by the dispersion relation in Eq. (5). Equation (21) can then be rewritten in terms of the flux tube radius as

$\frac{L_{\mathrm{D}}}{R}=2 \pi \frac{v_{\mathrm{Ai}}}{\omega R} \frac{\xi_{\mathrm{E}}}{m} \sqrt{\frac{2 \rho_{\mathrm{i}}}{\rho_{\mathrm{i}}+\rho_{\mathrm{e}}}}$,

where $\omega R / v_{\mathrm{Ai}}$ is a dimensionless frequency. The TT approximation means $\omega R / v_{\mathrm{Ai}} \ll 1$. Denoting this frequency as

$f=\frac{\omega R}{v_{\mathrm{Ai}}}$,

then (note the different definition of $f$ in Verth et al. 2010)

$\frac{L_{\mathrm{D}}}{R}=2 \pi \frac{\xi_{\mathrm{E}}}{m} \sqrt{\frac{2 \rho_{\mathrm{i}}}{\rho_{\mathrm{i}}+\rho_{\mathrm{e}}}} \frac{1}{f}$.

We refer to the relation between the damping length and frequency defined in Eq. (24) as the TGV relation for propagating kink waves. In what follows, it will become clear that the TGV relation is actually a very good approximation of the dependency of damping length on frequency for all relevant frequencies as illustrated in Fig. 2. The expression given in Eq. (24) has important consequences, as it shows that $L_{\mathrm{D}} / R$ is inversely proportional to $f$ for propagating waves; i.e., the damping length is inversely proportional to frequency, so that high frequency waves are damped on shorter spatial scales than their lower frequency counterparts. This means that for driven waves propagating upwards from the photosphere, each frequency has a different penetration height into the solar corona. Therefore, resonant absorption provides a natural filtering mechanism for broadband disturbances, e.g., like those observed by Tomczyk \& McIntosh (2009), with lower frequency waves being least affected by the damping process and propagating to higher heights in the solar corona.

\section{Spatial damping beyond the TT approximation}

The TT approximation is useful because it makes the dispersion relation analytically solvable. In Table 1 we list observed estimates of $k_{z} R$ for various MHD wave modes, and it can be seen that for many solar atmospheric waves, e.g., standing kink waves in coronal loops observed by Aschwanden et al. (2002), the TT approximation is reasonably valid. We have used the averaged values of loop radius and length. However, for kink waves in filament threads (Lin et al. 2009) or torsional Alfvén waves in chromospheric magnetic bright points (Jess et al. 2009) many have values of $k_{z} R \approx 1$. It is relatively straightforward to relax the TT approximation and to consider the effect of the finite tube radius on damping. 
Table 1. Estimated range of $k_{z} R$ in observed propagating $(\mathrm{P})$ and standing $(\mathrm{S}) \mathrm{MHD}$ wave modes.

\begin{tabular}{lcccc}
\hline \hline Reference & $\begin{array}{c}\text { MHD wave mode } \\
\text { interpretation of authors }\end{array}$ & $\begin{array}{c}\text { Width of } \\
\text { waveguide } \\
(\mathrm{Mm})\end{array}$ & $\begin{array}{c}\text { Wavelength } \\
(\mathrm{Mm})\end{array}$ & $k_{z} R$ \\
\hline 1 & Kink (S) & $5.9-11.5$ & $328-552$ & $0.03-0.11$ \\
2 & Alfvén (P) & 0.2 & $>4.0$ & $<0.16$ \\
3 & Kink or sausage waves (S) & 0.4 & $2.5-3.1$ & $0.41-0.5$ \\
4 & Kink or Alfvén waves (P) & 0.2 & $2.2-7.2$ & $0.17-0.58$ \\
5 & Torsional Alfvén (P) & 2.0 & $4.5-6.0$ & $1.04-1.39$ \\
6 & Kink (P) & $0.2-1.0$ & $3.4-12.9$ & $0.03-0.9$ \\
7 & Kink (P) & $0.43-0.66$ & $>250$ & $<0.12$ \\
8 & Alfvén (P) & 9.0 & $>393$ & $<0.07$ \\
9 & Kink or Alfvén waves (P) & 9.0 & $>180$ & $<0.16$ \\
\hline
\end{tabular}

References. (1) Aschwanden et al. (2002); (2) De Pontieu et al. (2007); (3) Fujimura \& Tsuneta (2009); (4) He et al. (2009); (5) Jess et al. (2009); (6) Lin et al. (2009); (7) Okamoto et al. (2007); (8) Tomczyk et al. (2007); (9) Tomczyk \& McIntosh (2009).

\subsection{Homogeneous magnetic cylinder}

The dispersion relation for a homogeneous magnetic cylinder is

$\rho_{\mathrm{i}}\left(\omega^{2}-\omega_{\mathrm{Ai}}^{2}\right)+\rho_{\mathrm{e}}\left(\omega^{2}-\omega_{\mathrm{Ae}}^{2}\right) F\left(\omega, k_{z}\right)=0$,

taking into account a finite radius, where

$$
\begin{aligned}
F\left(\omega, k_{z}\right) & =-\frac{k_{\mathrm{i}}}{k_{\mathrm{e}}} \frac{J_{m}^{\prime}\left(k_{\mathrm{i}} R\right) K_{m}\left(k_{\mathrm{e}} R\right)}{J_{m}\left(k_{\mathrm{i}} R\right) K_{m}^{\prime}\left(k_{\mathrm{e}} R\right)}, \\
k_{\mathrm{i}}^{2} & =\frac{\omega^{2}-\omega_{\mathrm{Ai}}^{2}}{v_{\mathrm{Ai}}^{2}}, \quad k_{\mathrm{e}}^{2}=-\frac{\omega^{2}-\omega_{\mathrm{Ae}}^{2}}{v_{\mathrm{Ae}}^{2}} .
\end{aligned}
$$

The function $F$ depends both on frequency $\omega$ and wave number $k_{z}$, taking that the tube has a finite width into account. Note that $F \equiv 1$ in the limit of a TT (the Bessel functions are approximated by their small arguments expressions), and we recover the dispersion relation given by Eq. (1). For a standing wave, $k_{z}$ is fixed and Eq. (25) is solved for $\omega$, while for a propagating wave, $\omega$ is fixed and Eq. (25) is solved for $k_{z}$. The solution to Eq. (25) corresponds again to the undamped kink wave. For a finite width tube the analytical solution to this equation would imply to use of additional terms in the asymptotic expansions of the Bessel functions, complicating matters. We simply solve Eq. (25) numerically in order to avoid these cumbersome calculations.

\subsection{Inhomogeneous magnetic cylinder}

When we add a thin non-uniform layer we obtain the complex dispersion relation

$$
\begin{aligned}
\rho_{\mathrm{i}}\left(\omega^{2}-\right. & \left.\omega_{\mathrm{Ai}}^{2}\right)+\rho_{\mathrm{e}}\left(\omega^{2}-\omega_{\mathrm{Ae}}^{2}\right) F\left(\omega, k_{z}\right)= \\
& \mathrm{i} \pi \frac{m / R}{\rho\left(r_{\mathrm{A}}\right)\left|\Delta_{\mathrm{A}}\right|} \rho_{\mathrm{i}}\left(\omega^{2}-\omega_{\mathrm{Ai}}^{2}\right) \rho_{\mathrm{e}}\left(\omega^{2}-\omega_{\mathrm{Ae}}^{2}\right) G\left(\omega, k_{z}\right),
\end{aligned}
$$

where

$G\left(\omega, k_{z}\right)=-\frac{m}{R} \frac{1}{k_{\mathrm{e}}} \frac{K_{m}\left(k_{\mathrm{e}} R\right)}{K_{m}^{\prime}\left(k_{\mathrm{e}} R\right)}$.

For the TT we can again use the asymptotic expansions of the Bessel function so that $G \equiv 1$, hence in the TT limit, Eq. (28) is simplified to Eq. (6). Equation (28) can be solved for a complex frequency $\omega$ with a specified real wavenumber $k_{*}$ or for a complex wavenumber $k_{z}$ for a specified real frequency $\omega_{*}$. By solving Eq. (28) in the case of a propagating wave with real frequency $\omega_{*}$ we find that the real part of complex $k_{z}$ is $k_{*}$ (we get again Eq. (25), $k_{*}$ is now different from Eq. (6)), while the imaginary part is given by

$\frac{k_{\mathrm{I}}}{k_{*}}=\frac{T}{N}$,

where

$$
\begin{aligned}
T= & \frac{\pi}{2} \frac{m}{R} \frac{\left(\rho_{\mathrm{i}}-\rho_{\mathrm{e}}\right)^{2}}{\left[\rho_{\mathrm{i}}+\rho_{\mathrm{e}} F\left(\omega_{*}, k_{*}\right)\right]\left[1+F\left(\omega_{*}, k_{*}\right)\right]^{2}} \\
& \times \frac{F\left(\omega_{*}, k_{*}\right) G\left(\omega_{*}, k_{*}\right)}{|\mathrm{d} \rho / \mathrm{d} r|_{r_{\mathrm{A}}}},
\end{aligned}
$$

and

$N=1+\left.\frac{k_{*}}{2} \frac{\rho_{\mathrm{i}}-\rho_{\mathrm{e}}}{\left[\rho_{\mathrm{i}}+\rho_{\mathrm{e}} F\left(\omega_{*}, k_{*}\right)\right]\left[1+F\left(\omega_{*}, k_{*}\right)\right]} \frac{\partial F}{\partial k}\right|_{\left(\omega_{*}, k_{*}\right)}$.

The expressions are slightly more complicated than in the TT approximation (see Eq. (10)), but once $k_{*}$ is known the different terms can be easily calculated. The corresponding damping per wavelength is

$\frac{L_{\mathrm{D}}}{\lambda}=\frac{1}{2 \pi} \frac{N}{T}$.

In the TT limit, i.e., when $F\left(\omega_{*}, k_{*}\right)$ and $G\left(\omega_{*}, k_{*}\right) \rightarrow 1$ and $\partial F / \partial k \rightarrow 0$, we recover Eq. (11). For a sinusoidal density profile we simply have to make use of Eq. (12) in (31). Now let us define

$\widetilde{N}=1-\left.\frac{\omega_{*}}{2} \frac{\rho_{\mathrm{i}}-\rho_{\mathrm{e}}}{\left[\rho_{\mathrm{i}}+\rho_{\mathrm{e}} F\left(\omega_{*}, k_{*}\right)\right]\left[1+F\left(\omega_{*}, k_{*}\right)\right]} \frac{\partial F}{\partial \omega}\right|_{\left(\omega_{*}, k_{*}\right)}$.

As in the previous section, we compared, the damping per wavelength for propagating waves with the damping per period, which in the non-TT approximation is given by

$\frac{\tau_{\mathrm{D}}}{P}=\frac{1}{2 \pi} \frac{\widetilde{N}}{T}$.

By Eqs. (33) and (35), there is a clear correspondence between the temporal and the spatial damping beyond the TT approximation. Furthermore, a general expression that relates the temporal damping of standing waves and the spatial damping of propagating waves can be derived following the analysis of Appendix A in Tagger et al. (1995). We can write the complex dispersion relation given by Eq. (28) as

$D_{\mathrm{R}}(\omega, k)+\mathrm{i} D_{\mathrm{I}}(\omega, k)=0$. 
To make it clear, in Eq. (36), $D_{\mathrm{R}}$ is equal to the LHS of Eq. (28) and $D_{\mathrm{I}}$ equal to minus the RHS of the equation. In the case of spatial damping, if $\omega_{*}$ and $k_{*}$ are the solutions of $D_{\mathrm{R}}(\omega, k)=0$ then it is easy to see by making a Taylor expansion around the solution that

$k_{\mathrm{I}}=-\left.\frac{D_{\mathrm{I}}}{\partial D_{\mathrm{R}} / \partial k}\right|_{\left(\omega_{*}, k_{*}\right)}$,

while for temporal damping

$\omega_{\mathrm{I}}=-\left.\frac{D_{\mathrm{I}}}{\partial D_{\mathrm{R}} / \partial \omega}\right|_{\left(\omega_{*}, k_{*}\right)}$.

Combining these two expressions we find that

$\frac{L_{\mathrm{D}}}{\lambda}=\frac{k_{*}}{\omega_{*}} \frac{\partial D_{\mathrm{R}} / \partial k}{\partial D_{\mathrm{R}} / \partial \omega} \frac{\tau_{\mathrm{D}}}{P}$

which is simply (see also Pascoe et al. 2010)

$\frac{L_{\mathrm{D}}}{\lambda}=\frac{v_{\mathrm{gr}}}{v_{\mathrm{ph}}} \frac{\tau_{\mathrm{D}}}{P}$

This result is valid when the damping is not too strong (see e.g., Tagger et al. 1995), an assumption that we have already made in deriving the damping per wavelength $\left(k_{\mathrm{I}} \ll k_{\mathrm{R}}\right)$. In the TT approximation, kink waves are weakly dispersive so that $v_{\mathrm{gr}} \approx v_{\mathrm{ph}}$ in agreement with the results found in Sect. 3, the damping per wavelength is thus exactly the same as the damping per period, but in the regime when the TT approximation is not applicable, the group speed can differ from the phase speed.

From the previous results, we can also derive alternative formulae for the damping per wavelength and damping per period in terms of the imaginary part of the dispersion relation,

$$
\begin{aligned}
\frac{L_{\mathrm{D}}}{\lambda}= & \frac{k_{*}}{2 \pi} \frac{1}{D_{\mathrm{I}}\left(\omega_{*}, k_{*}\right)}\left\{-2 k_{*}\left[\rho_{\mathrm{i}} v_{\mathrm{Ai}}^{2}+\rho_{\mathrm{e}} v_{\mathrm{Ae}}^{2} F\left(\omega_{*}, k_{*}\right)\right]\right. \\
& \left.+\left.\rho_{\mathrm{e}}\left(\omega_{*}^{2}-k_{*}^{2} v_{\mathrm{Ae}}^{2}\right) \frac{\partial F}{\partial k}\right|_{\left(\omega_{*}, k_{*}\right)}\right\},
\end{aligned}
$$

$$
\begin{aligned}
\frac{\tau_{\mathrm{D}}}{P}= & \frac{\omega_{*}}{2 \pi} \frac{1}{D_{\mathrm{I}}\left(\omega_{*}, k_{*}\right)} \\
& \times\left\{2 \omega_{*}\left[\rho_{\mathrm{i}}+\rho_{\mathrm{e}} F\left(\omega_{*}, k_{*}\right)\right]+\left.\rho_{\mathrm{e}}\left(\omega_{*}^{2}-k_{*}^{2} v_{\mathrm{Ae}}^{2}\right) \frac{\partial F}{\partial \omega}\right|_{\left(\omega_{*}, k_{*}\right)}\right\} .
\end{aligned}
$$

Using Eq. (40) we can identify in these expressions the terms related to the phase and group speed.

Next using the analytical results given in this section, we study how the damping per wavelength depends on the dimensionless frequency of the driver $f$, defined by Eq. (23), for particular cases (see Fig. 1). We see that the wider the layer, the more efficient the attenuation (smaller damping per wavelengths), in agreement with the analytical results in the TT approximation. For small $f$ the damping per wavelength tends to the TT value. The value of $L_{\mathrm{D}} / \lambda$ increases monotonically with $\omega$. The deviation with respect to the TT results is smaller for thick layers. In the TT approximation, which for propagating waves, is the low-frequency approximation, $L_{\mathrm{D}} / \lambda$ is independent of frequency, since this approximation does not take into account the variation of frequency; i.e., the frequency is only presumed to be low. The analytical results for $L_{\mathrm{D}} / \lambda$ beyond the TT approximation, take the dependence on frequency into account. The value of $L_{\mathrm{D}} / \lambda$ now undergoes a moderate increase when we move from

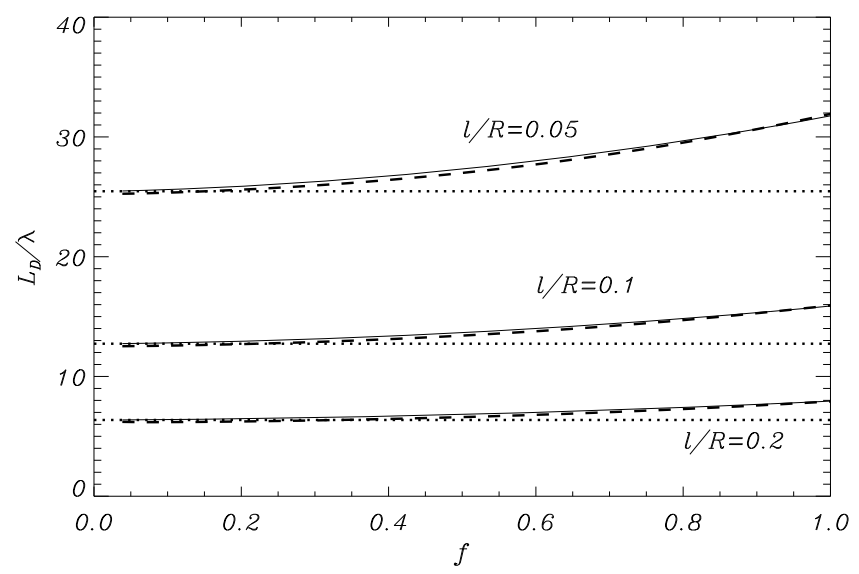

Fig. 1. Damping per wavelength as a function of the dimensionless frequency $\left(f=\omega R / v_{\mathrm{Ai}}\right)$ for three different widths of the inhomogeneous layers. The solid line corresponds to the analytical results, and the dashed line represents the full numerical solution of the resistive eigenvalue problem. The dotted line corresponds to the TT approximation, valid when $f \rightarrow 0$. In this plot $\rho_{\mathrm{i}} / \rho_{\mathrm{e}}=3$.

low to high frequencies. However, the really interesting quantity to calculate is the damping length itself. To make the dependence of $L_{\mathrm{D}}$ on frequency more explicit, we follow the same line of reasoning in Sect. 3.2 and rewrite Eq. (13) as

$\frac{L_{\mathrm{D}}}{\lambda}=\xi_{\mathrm{EW}}$,

where the quantity $\xi_{\mathrm{EW}}$ now depends on the parameters of the equilibrium model and the characteristics of the wave itself. Again $\lambda=2 \pi / k_{z}$ and $k_{z}$ is related to the frequency by the dispersion relation Eq. (25). Since we have abandoned the TT approximation, a simple analytical formula that relates $k_{z}$ to $\omega$ is not readily available, but we can always write

$k_{z} R=f \psi(f)$,

where $f$ is the dimensionless frequency defined in Eq. (23), and $\psi(f)$ is a function that we can determine numerically. As a result by implementing the function $\psi(f)$, we find that

$\frac{L_{\mathrm{D}}}{R}=2 \pi \frac{\xi_{\mathrm{EW}}}{\psi(f)} \frac{1}{f}$,

which in the low-frequency limit is equivalent to the TGV relation given by Eq. (24) for $m=1$; i.e.,

$\xi_{\mathrm{EW}} \rightarrow \xi_{\mathrm{E}} \quad$ and $\quad \psi \rightarrow \sqrt{\frac{\rho_{\mathrm{i}}+\rho_{\mathrm{e}}}{2 \rho_{\mathrm{i}}}}$

as $f \rightarrow 0$, where $\xi_{\mathrm{E}}$ is defined by Eq. (17). In Eq. (45) since $\psi(f)$ and $\xi_{\mathrm{EW}}$ are slowly varying functions of $f$, the main dependence of $L_{\mathrm{D}}$ on $f$ is contained in the factor $1 / f$, as in the low-frequency limit shown by Eq. (24). This is illustrated in Fig. 2, where $L_{\mathrm{D}} / R$ is plotted as a function of $f$ for several values of $l / R$. The dependence of the curves on $1 / f$ is very clear, and the non-TT and TT solutions tend to overlap in the limit of $f \rightarrow 0$, where both cases are accurately described by the TGV relation. Even for $f \rightarrow 1$ the TGV relation still describes the behaviour of the damping length with frequency quite well. Again, the potential of resonant absorption as a frequency filter is clearly demonstrated in Fig. 2, with high-frequency waves being damped on shorter spatial scales than low-frequency waves (for fixed $l / R$ ). 


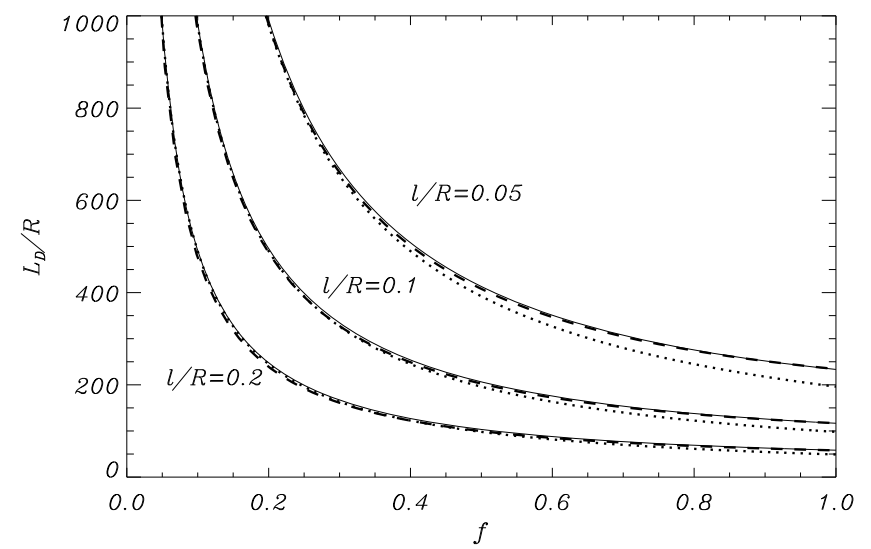

Fig. 2. Damping length normalised to the loop radius as a function of the dimensionless frequency $\left(f=\omega R / v_{\mathrm{Ai}}\right)$ for three different widths of the inhomogeneous layers. The solid line corresponds to the analytical results, the dashed line represents the full numerical solution of the resistive eigenvalue problem, and the dotted line corresponds to the TT approximation calculated using Eq. (24), valid when $f \rightarrow 0$. In this plot $\rho_{\mathrm{i}} / \rho_{\mathrm{e}}=3$.

\section{Resistive calculations}

The results based on TB approximation described in the previous sections are compared with the full resistive calculations using the same cylindrical tube model. The same approach as in Terradas et al. (2006) is used: i.e., the linearised MHD equations including magnetic diffusion are numerically solved using finite elements. This method allows us to calculate the complex eigenfrequencies of the quasimodes, which are independent of the value of the resistivity in the limit of large resistivity (see Poedts \& Kerner 1991).

The comparison with the analytical results is useful since there are no implicit assumptions about the TT or TB approximation in the resistive eigenvalue problem; i.e., the TT and TB approximations are not used. The resistive calculation applies to any frequency, whether it is small compared to $v_{\mathrm{Ai}} / R$ or not, and also to equilibrium models that have a thin non-uniform layer or are fully non-uniform. We have solved the eigenvalue problem (for $\omega$ ) and have used Eq. (40) to translate from temporal damping to spatial damping. By including resistivity, the eigenvalue problem for the wavenumber is more difficult to solve than the eigenvalue problem for the frequency. The results of the resistive calculations are shown in Figs. 1 and 2 where the damping per wavelength and the damping length are plotted as a function of the frequency of the driver. The agreement between the resistive computations and the analytical or semi-analytical methods is very good. The small differences are the result of assuming that the resonance is always located at $r=R$ in the analytical approximations, and we have calculated the derivative of the density at this position. This explains the small deviations from the resistive estimations. A more precise determination could be done by calculating the exact location of the resonance and then using a slightly modified version of Eq. (28), but since the analytical approximations that we have already derived are quite satisfactory, there is no pressing need to explore this further.

\section{Conclusions and discussion}

The spatial damping due to resonant absorption of driven kink waves has been investigated. The main conclusion of the work is that the damping length of propagating kink waves due to resonant absorption is a monotonically decreasing function of frequency. The TGV relation for kink waves was derived, demonstrating that for low frequencies the damping length is exactly inversely proportional to frequency. In the high-frequency range the TGV relation continues to be an excellent approximation of the actual dependency of the damping length on frequency. Certainly, for all physically relevant frequencies the dependency of damping length on frequency is accurately described by the TGV relation. This dependency means that resonant absorption is selective as it favours low-frequency waves and can efficiently remove high-frequency waves from a broad band spectrum of kink waves. This has high significance for solar atmospheric kink waves, since high-frequency waves will tend to lose more power than their low-frequency counterparts before reaching high altitudes in the solar corona, with the exact percentage power loss depending on the properties of the equilibrium, in particular the width of the non-uniform layer and steepness of the variation in the local Alfvén speed. With respect to mode conversion, the process of resonant absorption will cause the higher frequency waves to be attenuated more because the global kink mode will be converted into localised Alfvénic modes at lower heights. If the energy of these Alfvénic motions is eventually dissipated, then resonant absorption should produce a characteristic distribution of the energy as a function of height in the solar atmosphere. This could have important consequences with for the spatial distribution of wave heating in the solar atmosphere.

It has also been shown that spatial and temporal damping are basically equivalent. In the TT approximation, the damping per period and the damping per wavelength are exactly the same. The differences in these two quantities arise in the regime where the TT is not valid, but even in this situation it is easy to relate the spatial and the temporal damping rates through the group and phase speeds of the kink MHD waves. This allows us to translate the results from the temporally damped waves ( $\omega$ complex, $k$ real) to spatially attenuated waves ( $\omega$ real, $k$ complex) due to resonant absorption. This mechanism requires the frequency of the driver to be between the internal and the external Alfvén frequency of the tube. This might seem a very restrictive condition, but in fact it is just the opposite. In the driven problem, the frequency is fixed but the system chooses the proper wavelength (along the waveguide) to accommodate the kink mode in the tube. This kink mode generated at the base of the loop propagates upwards along the tube and at the same time is attenuated by the inhomogeneity at the tube boundary.

An interesting result is that both the damping length in the spatial problem and the damping time in the temporal problem are always smaller than in the TT approximation (when $f \rightarrow 0$ ), meaning that waves with short wavelengths or high frequencies are always more efficiently damped. The observations of standing kink waves observed with TRACE and for the propagating kink waves detected with the CoMP instrument are precisely in the regime where the TT is applicable, i.e., where the waves are less affected by resonant absorption. That overtones of standing kink waves and high-frequency propagating waves have proved difficult to detect may be a direct consequence of the filtering by resonant absorption. From a different perspective, we have also shown that the damping per wavelength (and the damping per period) has a weak dependence on the frequency.

It is necessary to point out that our results are based on a simple magnetic flux tube model, i.e., a straight cylinder, with no gravity and pressure. Curvature might produce some damping due to wave leakage and external resonances, while stratification 
might be important if the wavelength of the propagating kink modes is less than the typical density scale height of the solar corona. The effect of gas pressure should be small since the corona is expected to have very small beta. These issues need to be investigated in the future. The only inhomogeneity in our model is plasma density in the radial direction; however, this is a characteristic property of solar waveguides that is observed at all atmospheric heights, e.g., chromospheric magnetic bright points or coronal loops. Thus the theory of resonant damping of propagating kink waves due to radial plasma density inhomogeneity offers a natural explanation for the dissipation observed by, e.g., Tomczyk \& McIntosh (2009). However, a detailed comparison between the observations and the expected frequency-dependent response by resonant absorption is needed to quantify the precise spatial distribution of wave heating because this mechanism in the solar plasma, e.g., kink wave dissipation as a function of both frequency and height. This problem is addressed in Verth et al. (2010).

Acknowledgements. J.T. acknowledges the Universitat de les Illes Balears for a postdoctoral position and the funding provided under projects AYA2006-07637 (Spanish Ministerio de Educación y Ciencia). M.G. and G.V. acknowledge support from K. U. Leuven via GOA/2009-009. The authors thank the anonymous referee for useful comments that helped to improve the paper.

\section{References}

Andries, J., Arregui, I., \& Goossens, M. 2005a, ApJ, 624, L57

Andries, J., Goossens, M., Hollweg, J. V., Arregui, I., \& Van Doorsselaere, T. 2005b, A\&A, 430, 1109

Arregui, I., \& Ballester, J. L. 2010, Space Sci. Rev., accepted [arXiv: 1002.3489$]$

Arregui, I., Van Doorsselaere, T., Andries, J., Goossens, M., \& Kimpe, D. 2005, A\&A, 441, 361

Arregui, I., Andries, J., Van Doorsselaere, T., Goossens, M., \& Poedts, S. 2007, A\&A, 463, 333

Arregui, I., Terradas, J., Oliver, R., \& Ballester, J. L. 2008, ApJ, 682, L141

Aschwanden, M. J. 2005, ApJ, 634, L193

Aschwanden, M. J., Fletcher, L., Schrijver, C. J., \& Alexander, D. 1999, ApJ, 520,880

Aschwanden, M. J., de Pontieu, B., Schrijver, C. J., \& Title, A. M. 2002, Sol. Phys., 206, 99

Aschwanden, M. J., Nightingale, R. W., Andries, J., Goossens, M., \& Van Doorsselaere, T. 2003, ApJ, 598, 1375

Berghmans, D., \& de Bruyne, P. 1995, ApJ, 453, 495

Brady, C. S., \& Arber, T. D. 2005, A\&A, 438, 733

De Moortel, I., \& Brady, C. S. 2007, ApJ, 664, 1210

De Pontieu, B., Martens, P. C. H., \& Hudson, H. S. 2001, ApJ, 558, 859

De Pontieu, B., McIntosh, S. W., Carlsson, M., et al. 2007, Science, 318, 1574

Díaz, A. J., Oliver, R., Ballester, J. L., \& Roberts, B. 2004, A\&A, 424, 1055

Dymova, M. V., \& Ruderman, M. S. 2006, A\&A, 457, 1059

Edwin, P. M., \& Roberts, B. 1983, Sol. Phys., 88, 179

Erdélyi, R., \& Verth, G. 2007, A\&A, 462, 743

Fujimura, D., \& Tsuneta, S. 2009, ApJ, 702, 1443

Goossens, M. 2008, in IAU Symp., 247, 228
Goossens, M., Hollweg, J. V., \& Sakurai, T. 1992, Sol. Phys., 138, 233

Goossens, M., Ruderman, M. S., \& Hollweg, J. V. 1995, Sol. Phys., 157, 75

Goossens, M., Andries, J., \& Aschwanden, M. J. 2002, A\&A, 394, L39

Goossens, M., Andries, J., \& Arregui, I. 2006, Royal Soc. London Philos. Trans. Ser. A, 364, 433

Goossens, M., Arregui, I., Ballester, J. L., \& Wang, T. J. 2008, A\&A, 484, 851

Goossens, M., Terradas, J., Andries, J., Arregui, I., \& Ballester, J. L. 2009, A\&A, 503,213

He, J., Tu, C., Marsch, E., et al. 2009, A\&A, 497, 525

Heyvaerts, J., \& Priest, E. R. 1983, A\&A, 117, 220

Hollweg, J. V., \& Yang, G. 1988, J. Geophys. Res., 93, 5423

Ionson, J. A. 1978, ApJ, 226, 650

Jess, D. B., Mathioudakis, M., Erdélyi, R., et al. 2009, Science, 323, 1582

Lin, Y., Soler, R., Engvold, O., et al. 2009, ApJ, 704, 870

McLaughlin, J. A., \& Ofman, L. 2008, ApJ, 682, 1338

Morton, R. J., \& Erdélyi, R. 2009, ApJ, 707, 750

Nakariakov, V. M., Ofman, L., Deluca, E. E., Roberts, B., \& Davila, J. M. 1999, Science, 285, 862

Ofman, L. 2005, Adv. Space Res., 36, 1572

Ofman, L., \& Aschwanden, M. J. 2002, ApJ, 576, L153

Ofman, L., \& Davila, J. M. 1995, J. Geophys. Res., 100, 23427

Okamoto, T. J., Tsuneta, S., Berger, T. E., et al. 2007, Science, 318, 1577

Pascoe, D. J., Wright, A. N., \& De Moortel, I. 2010, ApJ, 711, 990

Poedts, S., \& Kerner, W. 1991, Phys. Rev. Lett., 66, 2871

Roberts, B. 2002, in Solar Variability: From Core to Outer Frontiers, ed. A. Wilson, ESA SP, 506, 481

Roberts, B., Edwin, P. M., \& Benz, A. O. 1984, ApJ, 279, 857

Ruderman, M. S., \& Erdélyi, R. 2009, Space Sci. Rev., 149, 199

Ruderman, M. S., \& Roberts, B. 2002, ApJ, 577, 475

Ruderman, M. S., Verth, G., \& Erdélyi, R. 2008, ApJ, 686, 694

Sakurai, T., Goossens, M., \& Hollweg, J. V. 1991, Sol. Phys., 133, 227

Selwa, M., Murawski, K., Solanki, S. K., Wang, T. J., \& Tóth, G. 2005, A\&A, 440,385

Selwa, M., Murawski, K., Solanki, S. K., \& Wang, T. J. 2007, A\&A, 462, 1127

Smith, J. M., Roberts, B., \& Oliver, R. 1997, A\&A, 317, 752

Soler, R., Oliver, R., \& Ballester, J. L. 2009a, ApJ, 707, 662

Soler, R., Oliver, R., Ballester, J. L., \& Goossens, M. 2009b, ApJ, 695, L166

Steinolfson, R. S., \& Davila, J. M. 1993, ApJ, 415, 354

Tagger, M., Falgarone, E., \& Shukurov, A. 1995, A\&A, 299, 940

Terradas, J. 2009, Space Sci. Rev., 149, 255

Terradas, J., Oliver, R., \& Ballester, J. L. 2006, ApJ, 642, 533

Terradas, J., Arregui, I., Oliver, R., et al. 2008, ApJ, 679, 1611

Tirry, W. J., \& Goossens, M. 1996, ApJ, 471, 501

Tirry, W. J., Berghmans, D., \& Goossens, M. 1997, A\&A, 322, 329

Tirry, W. J., Cadez, V. M., Erdélyi, R., \& Goossens, M. 1998, A\&A, 332, 786

Tomczyk, S., \& McIntosh, S. W. 2009, ApJ, 697, 1384

Tomczyk, S., McIntosh, S. W., Keil, S. L., et al. 2007, Science, 317, 1192

Uchida, Y. 1970, PASJ, 22, 341

Van Doorsselaere, T., Brady, C. S., Verwichte, E., \& Nakariakov, V. M. 2008, A\&A, 491, L9

Van Doorsselaere, T., Birtill, D. C. C., \& Evans, G. R. 2009, A\&A, 508, 1485

Vasheghani Farahani, S., Van Doorsselaere, T., Verwichte, E., \& Nakariakov, V. M. 2009, A\&A, 498, L29

Verth, G., \& Erdélyi, R. 2008, A\&A, 486, 1015

Verth, G., Van Doorsselaere, T., Erdélyi, R., \& Goossens, M. 2007, A\&A, 475, 341

Verth, G., Erdélyi, R., \& Jess, D. B. 2008, ApJ, 687, L45

Verth, G., Terradas, J., \& Goossens, M. 2010, ApJ, 718, L102

Verwichte, E., Nakariakov, V. M., Ofman, L., \& Deluca, E. E. 2004, Sol. Phys., 223, 77

Verwichte, E., Foullon, C., \& Nakariakov, V. M. 2006, A\&A, 446, 1139 